\title{
186 - Improving the Speed and Accuracy of Large-scale Scanning Transmission Electron Microscopy (STEM) Electron Scattering Simulations
}

Colin Ophus ${ }^{1}$, Hamish Brown ${ }^{1}$, Luis Rangel Dacosta ${ }^{1}$, Philipp Pelz ${ }^{2}$, Jonathan Schwartz ${ }^{3}$, Reed Yalisove $^{3}$, Robert Hovden ${ }^{3}$, Jim Ciston ${ }^{1}$ and Benjamin Savitzky ${ }^{4}$

${ }^{1}$ Lawrence Berkeley National Laboratory, Berkeley, California, United States, ${ }^{2}$ University of California Berkeley, Berkeley, California, United States, ${ }^{3}$ University of Michigan, Ann Arbor, Michigan, United States, ${ }^{4}$ National Center for Electron Microscopy (NCEM), Molecular Foundry, Lawrence Berkeley National Laboratory, Berkeley, California, United States

In a scanning transmission electron microscopy (STEM) experiment, a converged electron probe is typically scanned across a sample in a 2D grid of probe positions. At each STEM probe position, various signal channels can be recorded. These include imaging modes concerned primarily with electron scattering, such as annular bright field (ABF), annular dark field (ADF), or segmented-detector differential phase contrast (DPC), where we use a few monolithic detectors that measure the number of electrons which are scattered to various angular ranges to produce 2D image outputs. We can also perform spectroscopy, by either electron energy loss spectroscopy (EELS) on the forward scattered inelastic electrons, or by energy dispersive X-ray (EDX) spectroscopy where X-rays produced by the STEM probe interacting with the sample is used to perform chemical mapping, both of which produce 3D datasets. And finally, modern high-speed electron detectors also allow us to measure a full 2D image of the forwarddiffracted STEM probe at each probe position, producing a 4D dataset often referred to as a 4D-STEM experiment [1]. In many of these experiments, performing a quantitative analysis of the results requires us to perform electron scattering simulations for every position of the scanned electron probe.

STEM simulations for the 2D, 3D or 4D experiments described above are typically performed using the multislice method [2]. However, these simulations have traditionally required very long compute times, due to the fact that a full quantum-mechanical scattering simulation must be performed for each new probe position. Recently, we have developed the plane-wave reciprocal-space interpolated scattering matrix (PRISM) algorithm which can offer dramatic reductions in the time required to perform STEM simulations [3]. We have implemented multi-CPU and multi-GPU implementations of multislice and PRISM in the Prismatic simulation program [4], and recently extended the PRISM method to inelastic scattering simulations such as EELS [5]. However, while the PRISM method can offer orders-ofmagnitude speed-up, it can potentially also reduce the accuracy of a STEM simulation. In this talk, we describe various methods to further improve both the speed and accuracy of STEM simulations, using both the multislice and PRISM methods.

Figure 1 shows results from two of our improved simulation methods. Figure 1a shows the atomic coordinates used as input for these examples, and Figure 1b shows the projected potential along the beam direction. Figure 1c compares side views of the calculated projected potential using both a 2D lookup table method, and an improved 3D lookup table method. This method can quickly and accurately calculate a more accurate slicing of the atomic potentials in 3D, include thermal vibrations along the beam direction, and include subpixel atomic positions with higher accuracy. Figure 1d shows a STEM simulation using PRISM. For this simulation, an interpolation factor of $f=10$ produces an accurate simulation, while $f=$ 20 produces large errors due to cropping of the STEM probe. We have developed an extension of the PRISM method where the S-matrix is re-focused, to reduce the spread of the STEM probe and provide a 
significantly more accurate simulation. Using S-matrix refocusing, simulations can be run much faster by utilizing larger interpolation factors without sacrificing accuracy. In this talk, we will also describe several other newly developed methods including real space interpolation for faster bright field simulations, real space cropping and tiling methods for multislice simulations, and another algorithm called plane-wave reciprocal-space interpolated multislice (PRIM) for unit cell STEM calculations [6].
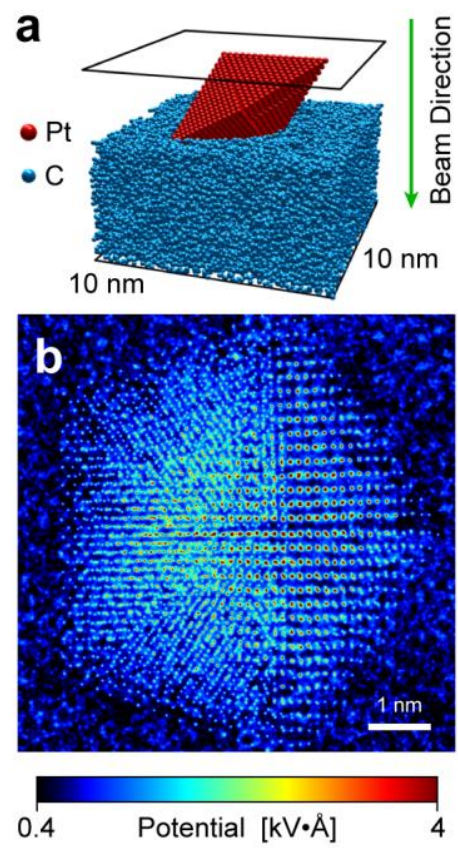

d Single probe position in center
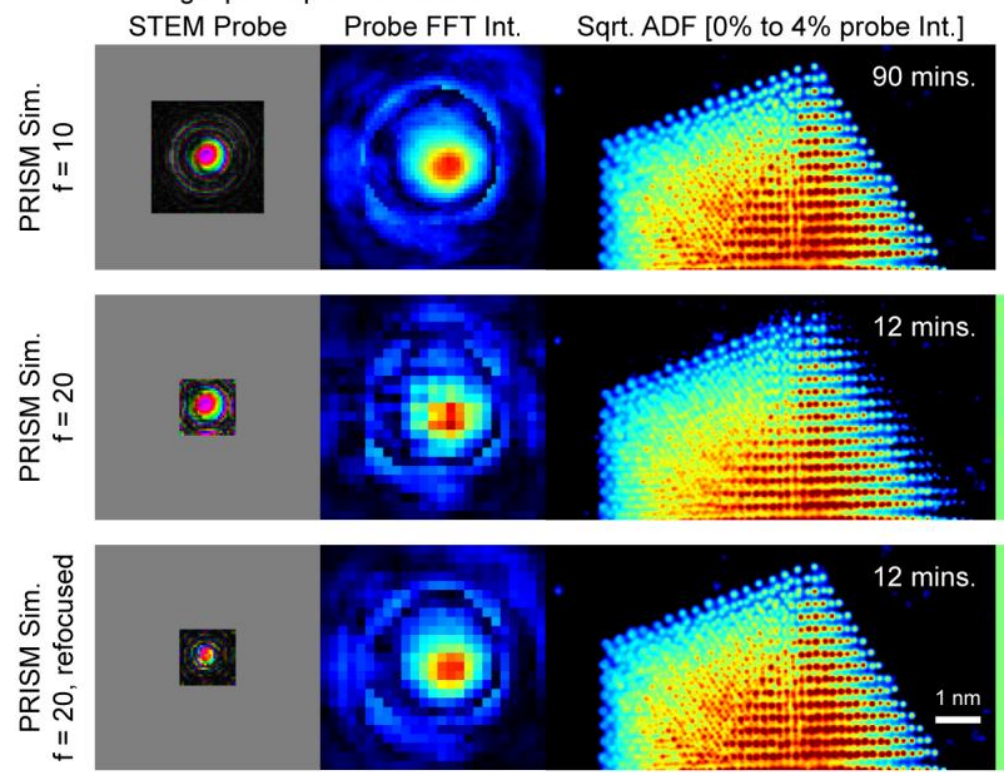

C Side view of projected potential, calculated with look-up table 2D projected potential

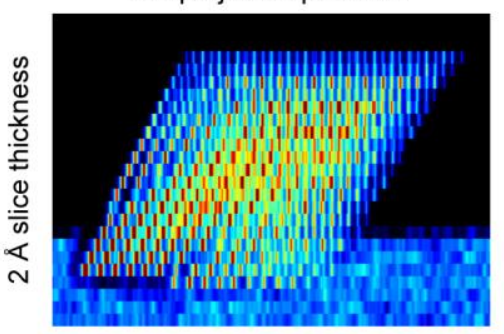

3D projected potential
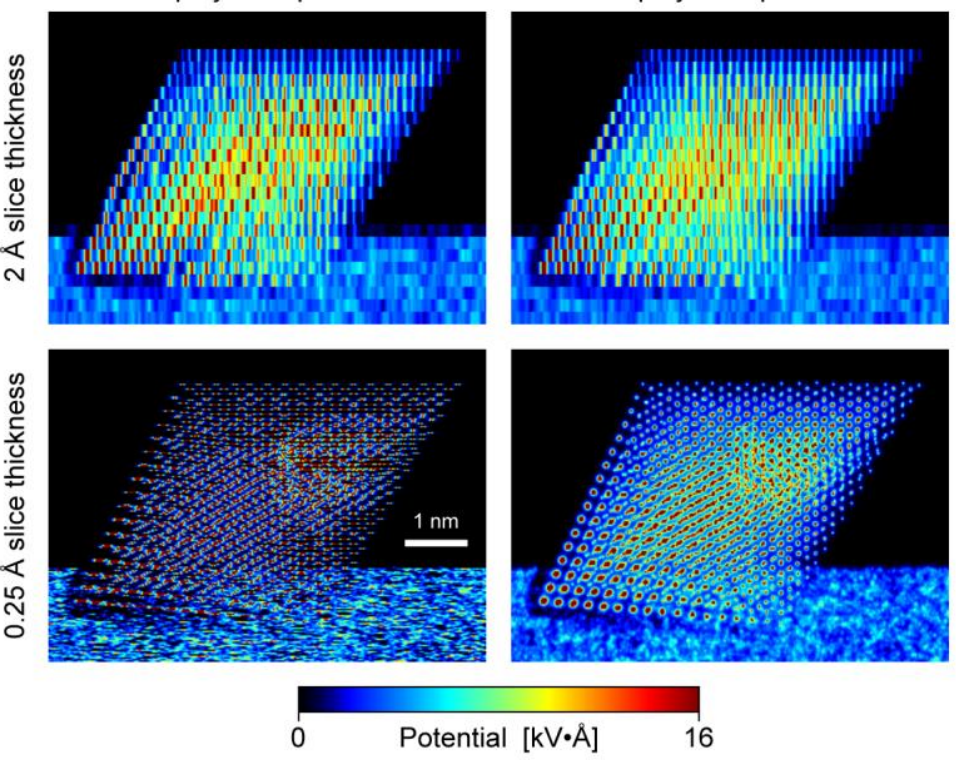

Multislice Simulation

STEM Probe Probe FFT Int

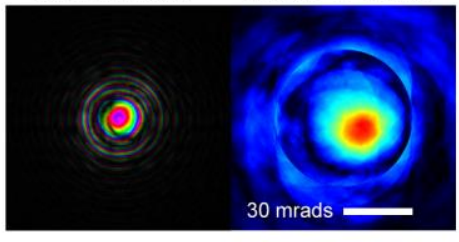

ADF Error [-2\% to $2 \%$ probe Int.]

Figure 1. Examples of improved STEM simulations. (a) Atomic coordinates, used to calculate (b) projected potential along the beam direction. (c) Side view of calculated projected potentials, comparing the 2D and the newly-developed 3D lookup table method. (d) ADF STEM simulation using PRISM with $\mathrm{f}=10, \mathrm{f}=20$, and the newly developed $\mathrm{f}=20$ refocused PRISM method. Calculation times inset. 


\section{References}

[1] C Ophus, Microscopy and Microanalysis 25, 563 (2019).

[2] JM Cowley, and AF Moodie, Acta Crystallographica 10, 609-619 (1957).

[3] C Ophus, Advanced Structural and Chemical Imaging 3 (13), 11 (2017).

[4] AJ Pryor Jr., C Ophus, and J Miao, Advanced structural and chemical imaging 3 (15), 15 (2017).

[5] HG Brown, J Ciston, and C Ophus, Physical Review Research 1 (3), 033186 (2019).

[6] Work at the Molecular Foundry was supported by the Office of Science, Office of Basic Energy Sciences, of the U.S. Department of Energy under Contract No. DE-AC02-05CH11231. CO acknowledges additional support from the U.S. Department of Energy Early Career Research Award Program. HGB and JC acknowledge additional support from the Presidential Early Career Award for Scientists and Engineers (PECASE) through the U.S. Department of Energy. PP acknowledges support from the STROBE NSF Science and Technology Center on Real-Time Functional Imaging. BHS acknowledges support from the Toyota Research Institute. 